\title{
Socioeconomia e etnoconhecimento de pescadores artesanais da comunidade do Cajueiro, distrito de Mosqueiro, Amazônia Oriental
}

\author{
Socioeconomy and ethnocognition of artisanal fishermen from the cashew community, Mosqueiro \\ district, eastern Amazon \\ Socioeconomía y etnocognición de pescadores artesanales de la comunidad del anacaro, distrito de \\ Mosqueiro, Amazonia oriental
}

\begin{abstract}
Resumo
A região de Mosqueiro-Belém do Pará, apresenta uma relação de dependência direta da comunidade com os ecossistemas dessa região, dentre as quais, a pesca é uma das principais atividades econômicas. O presente trabalho tem por objetivo avaliar a pesca da comunidade do Cajueiro, com ênfase nos aspectos socioeconômicos dos pescadores e o conhecimento etnoecológico das espécies. Através de entrevistas semiestruturadas foi possível observar que a comunidade pesqueira, as mulheres apresentam média de 36 anos enquanto os homens apresentaram 43 anos, dentre eles $70 \%$ dos pescadores são associados na colônia de pesca e as relações de trabalho são familiares, a atividade pesqueira é desenvolvida principalmente por homens devido os fatores de risco, as mulheres por sua vez participam principalmente da pesca do camarão, revenda do pescado, confecção e reparos das redes de pesca. O nível de escolaridade é baixo, com $90 \%$ com ensino fundamental incompleto e apenas $10 \%$ possuem fundamental completo. A comunidade utiliza barco de pequeno porte e a rede de emalhe. O conhecimento dos pescadores sobre o comportamento das espécies capturadas demonstra a relação com a percepção nas mudanças sazonais bem como o comportamento ecológico das espécies. Essas informações são uteis para o desenvolvimento de estratégias e planos de governo nos espectros ambientais e sociais, visto que é necessário um plano de manejo para as espécies capturadas devido ao intenso esforço de pesca. Quanto ao social é necessário traçar metas para o desenvolvimento escolar, além de promover o incentivo para a valorização das mulheres na pesca.
\end{abstract}

Palavras-chave: Conhecimento ecológico local; Recursos pesqueiros; Região amazônica. 


\begin{abstract}
The Mosqueiro-Belém do Pará region has a relationship of direct dependence on the community with the ecosystems of that region, among which, fishing is one of the main economic activities. The present work aims to evaluate the fishing of the Cajueiro community, with an emphasis on the socioeconomic aspects of the fishermen and the ethnoecological knowledge of the species. Through semi-structured interviews, it was possible to observe that the fishing community, women are on average 36 years old, while men are 43 years old, among them $70 \%$ of the fishermen are members of the fishing colony and the work relationships are familiar, the fishing activity is developed mainly by men due to risk factors, women in turn participate mainly in shrimp fishing, reselling fish, making and repairing fishing nets. The level of education is low, with $90 \%$ with incomplete elementary school and only $10 \%$ have complete elementary school. The community uses a small boat and a gillnet. Fishermen's knowledge of the behavior of the species caught demonstrates the relationship with the perception of seasonal changes as well as the ecological behavior of the species. This information is useful for the development of government strategies and plans in the environmental and social spectra, since a management plan is necessary for the species caught due to the intense fishing effort. As for the social, it is necessary to set goals for school development, in addition to promoting the incentive for the valorization of women in fishing.
\end{abstract}

Keywords: Local ecological knowledge; Fishing resources; Amazon region.

\title{
Resumen
}

La región Mosqueiro-Belém do Pará tiene una relación de dependencia directa de la comunidad con los ecosistemas de esa región, entre los cuales, la pesca es una de las principales actividades económicas. El presente trabajo tiene como objetivo evaluar la pesca de la comunidad de Cajueiro, con énfasis en los aspectos socioeconómicos de los pescadores y el conocimiento etnoecológico de la especie. A través de entrevistas semiestructuradas, se pudo observar que en la comunidad pesquera, las mujeres tienen en promedio 36 años, mientras que los hombres tienen 43 años, entre ellos el $70 \%$ de los pescadores son miembros de la colonia pesquera y las relaciones laborales son familiares, la actividad pesquera es Desarrollado principalmente por hombres debido a factores de riesgo, las mujeres a su vez participan principalmente en la pesca de camarón, reventa de pescado, confección y reparación de redes de pesca. El nivel de educación es bajo, con un $90 \%$ con primaria incompleta y solo un $10 \%$ con primaria completa. La comunidad utiliza un bote pequeño y una red de enmalle. El conocimiento de los pescadores sobre el comportamiento de las especies capturadas demuestra la relación con la percepción de los cambios estacionales y el comportamiento ecológico de la especie. Esta información es útil para el desarrollo de estrategias y planes gubernamentales en el espectro ambiental y social, ya que es necesario un plan de manejo para las especies capturadas debido al intenso esfuerzo pesquero. En cuanto a lo social, es necesario fijar metas de desarrollo escolar, además de promover el incentivo para la valorización de la mujer en la pesca.

Palabras clave: Conocimiento ecológico local; Recursos pesqueros; Región amazónica.

\section{Introdução}

Importantes aliados da gestão, os estudos econômicos disponibilizam parâmetros, teorias e técnicas de estudo que relaciona os fatores de produção e sociedade, analisando as questões de que, quando, quanto e para quem produzir, aonde suas respostas vêm das observações, do controle e acompanhamento de comportamentos e tendências, possivelmente representados por indicadores (Araújo et al, 2015).

A pesca é uma atividade de elevada importância social e econômica, sendo a pesca artesanal realizada em parceria ou de maneira isolada, utilizando apetrechos simples de captura (Ramires et al, 2012), essa prática apresenta dependência direta dos recursos naturais o que gradualmente gera conhecimentos sobre os animais capturados assim como os processos ambientais (McQueen, 1989).

Begossi (2010) ressalta que os pescadores artesanais podem contribuir às pesquisas em ecologia e etnoecologia através do compartilhamento das técnicas de manejo e captura do pescado, além do compartilhamento do conhecimento ambiental.

Mourão e Nordi (2006) definem a etnoecologia como uma linha de pesquisa científica transdisciplinar conciliando os conhecimentos científicos, religiosos, sentimentais e comportamentais das interações entre o homem e a natureza. Bahuchet, 1992, acrescenta que a etnoictiologia por sua vez consiste num ramo específico dos conhecimentos sobre o pescado e o uso dos mesmos.

Os estudos envolvendo os conhecimentos ecológicos, também conhecidas por saberes tradicionais, tem por premissa 
entender a interação entre as comunidades tradicionais e a maneira como fazem uso dos recursos naturais por meio do manejo, captura, comercialização, classificação e identificação da fauna e da flora (Marques, 1995; Mourão \& Nordi, 2006).

Considerando o cenário socioeconômico da região amazônica, fica evidente a necessidade de pesquisas no âmbito socioeconômico e etnoictiologico para a região. Desta forma, o objetivo do estudo foi de avaliar as características socioeconômicas e etnoictiologica da pesca artesanal da comunidade do Cajueiro, distrito de Mosqueiro, gerando dados que possam ser elencados na gestão.

\section{Metodologia}

\section{Área de estudo}

A Ilha de Mosqueiro é um distrito administrativo de Belém, situa-se na porção leste do estuário Guajarino, contida na região metropolitana de Belém (Sales et al., 2018). O trabalho foi desenvolvido, na comunidade que reside no entorno do rio Cajueiro (Figura 1).

Figura 1. Localização da comunidade do Cajueiro, Mosqueiro, Pará-Brasil.

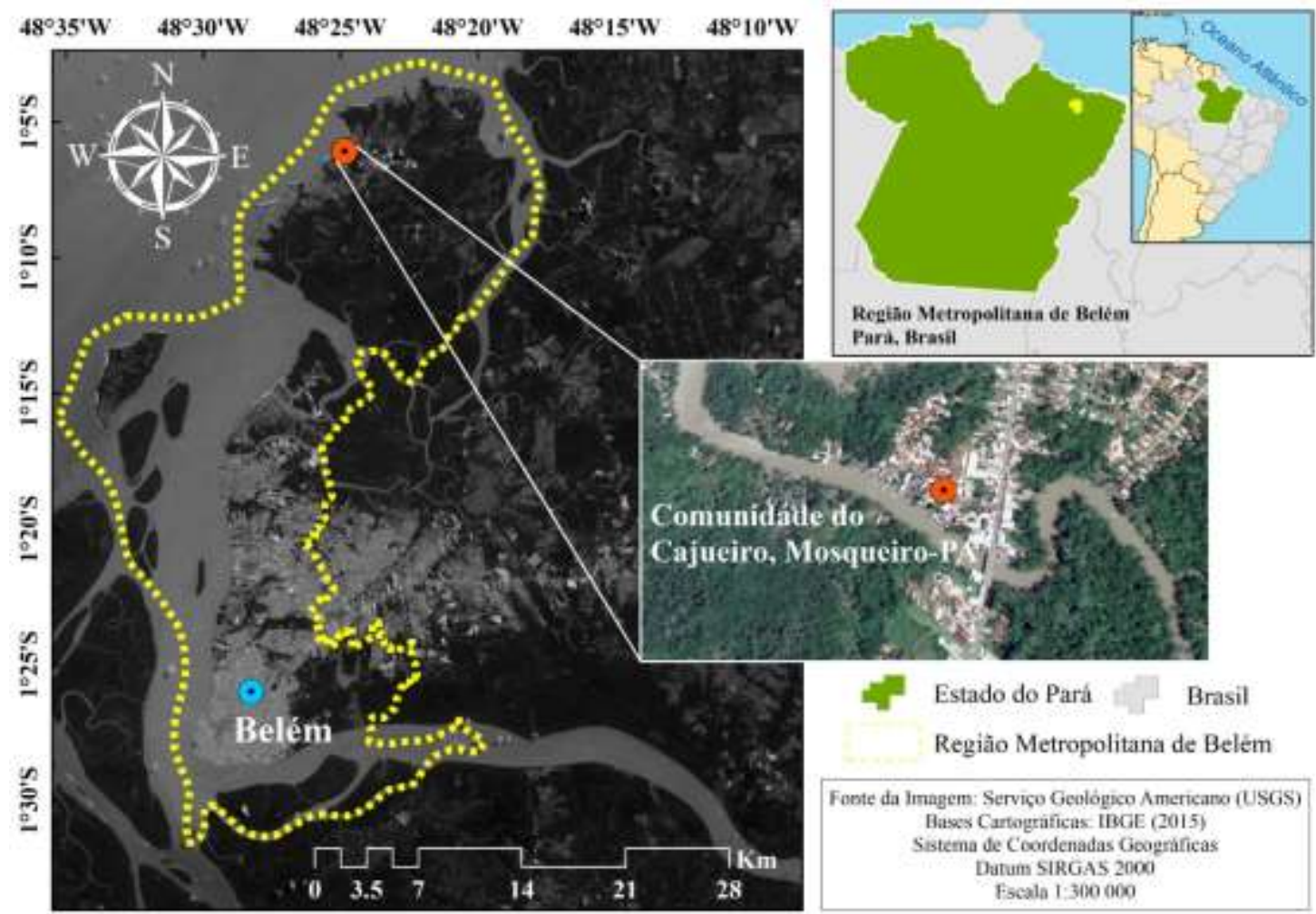

Fonte: Autores (2021).

\section{Coleta de dados e análises}

Utilizou-se classificação de Prodanov \& Freitas (2013), no qual, do ponto de vista da natureza a pesquisa classifica-se como pesquisa aplicada. Em relação aos seus objetivos foi classificada como descritiva. Já para os procedimentos técnicos foi classificada como Levantamento (survey). A metodologia aplicada foi baseada na coleta de dados por meio de entrevistas com formulários semiestruturados, aplicados aos entrevistados (pessoas que utilizam a pesca como sua atividade principal), entre os meses de abril a junho de 2013.

A forma de seleção dos entrevistados, se deu com base no método "bola de neve", no qual um entrevistado indica um próximo entrevistado e assim sucessivamente (Bailey, 1982). 
Foram aplicados 100 questionários semiestruturados, com o intuito de analisar a extensão técnica de produção, a qual envolve os apetrechos utilizados para atividade pesqueira e dados sobre o conhecimento dos pescadores acerca dos recursos ictiofaunísticos.

As etnoespécies foram nomeadas pelos pescadores e no momento do desembarque foram identificadas de acordo com a chave taxonômica de Espirito-Santo et al. (2005). A nomenclatura dos táxons seguiu o padrão internacional, utilizando-se a base de dados online da FishBase (Froese \& Pauly, 2019).

As informações coletadas foram digitalizadas, sendo posteriormente analisadas com estatística descritiva para os dados quantitativos. Os dados qualitativos obtidos, foram organizados em categorias descritivas, sendo a análise das respostas realizada de forma relacionada com os padrões encontrados nas literaturas sobre as espécies e os métodos de captura (Brag a \& Rebêlo, 2017; Silva \& Braga 2016; Pereira et. al, 2018).

\section{Resultados e Discussão}

\section{Perfil dos pescadores artesanais}

A pesca artesanal é uma das atividades tradicionais desenvolvidas na região amazônica, no entanto, cada comunidade pesqueira possui particularidades que definem as formas de manejo dos recursos naturais e do ambiente em que ocupam (Santos \& Santos, 2005). Para entendermos melhor o contexto da pesca artesanal na comunidade do Cajueiro-PA, é importante ressaltar que há pessoas que vivem direta ou indiretamente da pesca, é um dos pontos de desembarque pesqueiro da frota artesanal atuante no estuário amazônico devido à proximidade com Belém.

Os pescadores apresentam em média de 36 anos para as mulheres e 43 anos para os homens, com famílias constituídas em média de três filhos, onde $70 \%$ dos pescadores são associados a colônia de pesca Z9 de Mosqueiro, bem como, relações de trabalho familiares. Na comunidade do Cajueiro a atividade de pesca é desenvolvida principalmente por homens, ressaltando que a pesca chamada de em "alto mar" apresenta alguns fatores de risco, de acordo com o relatado por pescadores que destacam a atividade perigosa, insalubre e sem garantias econômicas, estes fatores contribuem para a ausência das mulheres nesse tipo de pescaria.

As mulheres representam 10\% dos entrevistados além de serem responsáveis pelas atividades domésticas, participam principalmente da pesca do camarão-da-amazônia (Macrobrachium amazonicum), esta modalidade de captura consiste em colocar armadilhas denominadas de matapi, fixadas em estacas de madeira próximas as suas residências na beira do igarapé do cajueiro, realizam a confecção e os reparos das redes de pesca além de trabalharem com a revenda do pescado no comércio localizado na ponte da comunidade. Conforme Maneschy et al. (2012), o papel que as mulheres desempenham na pesca reside na construção cultural da divisão de trabalho, pois essa divisão é muito marcada e forte nas comunidades pesqueiras, para analisar estas relações entre os gêneros, faz-se necessário considerar os atores envolvidos e as naturalizações relacionadas às representações de gênero e a identidade social.

A participação das mulheres na pesca, ainda não é valorizada mesmo que a atividade represente uma parcela da renda familiar, o maior valor é atribuído a pesca dos homens, a renda das mulheres é caracterizada como ajuda, como uma forma de submissão a dependência dos maridos (Walter et al., 2012; Fonseca et al., 2016). Santos et al. (2018), acrescenta que, a figura feminina destaca-se apenas no final da atividade, quando está relacionado ao beneficiamento do pescado e confecção de apetrechos, ressaltando a participação direta e indireta na atividade pesqueira.

O nível de escolaridade é baixo, com $87 \%$ dos homens e 3\% das mulheres com ensino fundamental incompleto, apenas $10 \%$ possuem fundamental completo (7\% mulheres e 3\% homens), o baixo nível escolar pode ser influenciado pela dificuldade de conciliar o trabalho com os estudos, que desde muito cedo começaram a pescar para ajudar a família na atividade. 
Conforme Alencar \& Maia (2011), o baixo índice de escolaridade entre os pescadores é comum na região Norte, possuindo apenas o ensino fundamental incompleto. Dentre outros aspectos o baixo grau de instrução de pescadores artesanais remete a tempos em que o acesso à escola era mais difícil, além da incompatibilidade de horário entre as atividades de pesca e o estudo, bem como o elevado esforço físico, resultando na evasão escolar (Borcem et al., 2011; Ramires et al., 2012).

Em contraste a realidade escolar dos pescadores da comunidade, os entrevistados relataram que seus filhos frequentam a escola, demonstrando o interesse em maior grau de escolaridade como uma alternativa para obter outra profissão. Hoje se observa na região da comunidade, que cada vez mais os filhos dos pescadores têm diminuído o interesse em praticar a atividade de pesca, segundo os relatos estes fatores estão associados há um maior acesso aos meios de informação.

Neste aspecto, frisa-se que a pesca artesanal tem início na infância, cujos conhecimentos sobre a atividade são repassados, evidenciando uma tradição familiar com intuito de que seus descendentes permaneçam na atividade pesqueira, no entanto o acesso facilitado à escola nos dias atuais influencia diretamente nas relações de trabalho e nas familiares, contribuindo para que os jovens busquem atividades mais rentáveis, afastando-se do ambiente pesqueiro (Garcez \& ShanchezBotero, 2005; Borcem et al., 2011).

Na comunidade as casas encontram-se principalmente na beira do rio, este tipo de ocupação urbana é comum na região amazônica, devido à retirada de parte da cobertura vegetal da área de várzea, bem como, aos assentamentos humanos nas áreas de terra, nas proximidades o rio encontra-se em estado de assoreamento. A área é habitada com construções desordenadas de casas em alvenarias (22\%) e madeiras (78\%), com o sistema de abastecimento d'água precário e usufruem, predominantemente, da assistência pública de saúde.

Por ser um entreposto pesqueiro, os pescadores comercializam o pescado próximo ao trapiche onde o produto desembarcado é adquirido por atravessadores, sendo comercializado nas bancas montadas na ponte. Todos os resíduos de peixe (resto de evisceração), descartes e aparas são descartados diretamente na água, poluindo e contaminando a mesma que é utilizado para a lavagem do pescado e outras atividades de limpeza doméstica. Silva et al. (2017), relata que, os portos presentes ao longo do rio e localizados próximos à ponte são os principais pontos de escoamento do pescado, estes pequenos portos são privados pertencendo a algumas famílias de relativo poder aquisitivo e que possuem embarcações e pequenos comércios na região.

Quanto à naturalidade dos entrevistados estes são principalmente de Mosqueiro, sendo que os municípios de Abaetetuba e Barcarena tiveram uma boa representatividade, estes municípios são próximos e com forte tradição na pesca, evidenciando as migrações ocorridas para a comunidade (Figura 2). 
Figura 2. Percentual da naturalidade dos entrevistados na comunidade do Cajueiro, Mosqueiro, Pará-Brasil.

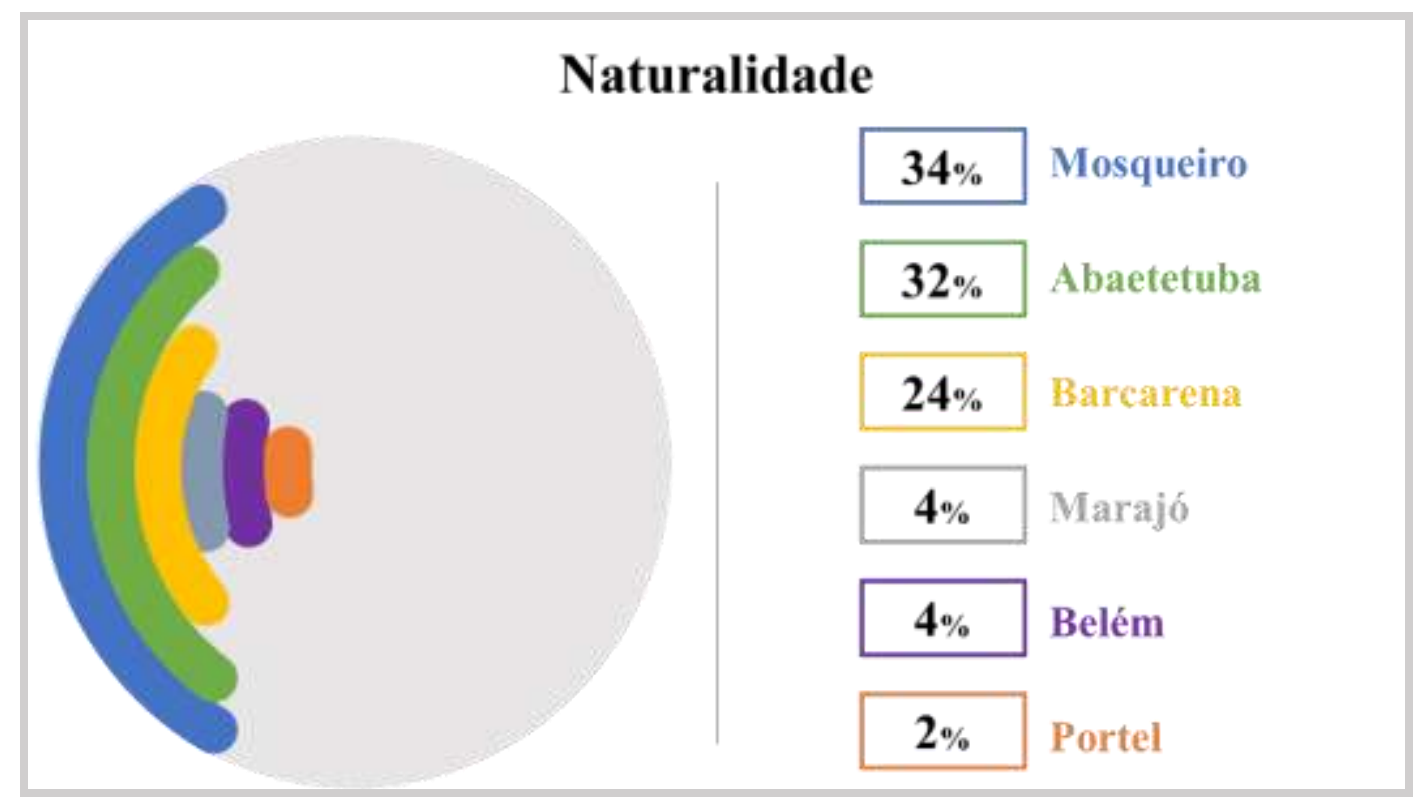

Fonte: Autores (2021).

\section{Aspectos tecnológicos e biológicos da pesca artesanal}

A comunidade utiliza barco de pequeno porte que é a embarcação do casco de madeira, com casaria, movida a motor, utilizando como único apetrecho a rede de emalhe que é confeccionada em nylon mono-filamento com 50, 60 e 70mm entre nós adjacentes e medem em média 1.100 metros para a captura de peixes diversos. A captura ocorre pela retenção dos peixes na malha da rede, também denominada de rede de espera, que possui formato retangular, estendidas nos pontos de passagem de cardumes.

Silva et al. (2017), caracteriza a pesca da comunidade do Cajueiro como uma atividade do tipo artesanal, com embarcações do tipo pequeno e de médio porte, com capacidade para transportar entre $80 \mathrm{~kg}$ a $100 \mathrm{~kg}$ de pescado, variando conforme o ambiente e as condições de pescaria, e embarcações com motor à diesel.

Essa arte de pesca é utilizada pelos moradores no canal dos rios, furos ou baias e ocorre ao longo de todo o ano, porém os meses de junho a dezembro são considerados pelos pescadores o mês de safra, ou seja, é o período de migração dos peixes, os locais de pesca são definidos através da observação do deslocamento e formação de cardumes.

O segundo semestre é também conhecido por período de seca, quando as chuvas diminuem e a vazão de água doce diminui aumentando assim a influência de água salgada na região (Martorano et al., 1993), este período influencia na duração das viagens, é quando ocorre à pesca do vai e vem, onde os pescadores saem pela manhã e retornam à tarde, o horário de saída e retorno das embarcações estão acondicionadas ao ponto mais alto da maré (maré alta) pois facilita o movimento das embarcações pelo igarapé do cajueiro.

Em relação aos ambientes de pesca existentes durante o período do inverno, muitos pescadores se deslocam para locais mais afastados da ilha, com viagens em torno de 12 horas para se afastar da costa, os locais citados pelos pescadores foram: a costa de Soure no Marajó, a costa da cidade de Vigia e Colares, o tempo da pesca é de 10 a 16 dias (Figura 3 ). 
Figura 3. Localização das áreas de pesca artesanal utilizadas pelos pescadores da comunidade do Cajueiro, Mosqueiro, ParáBrasil.

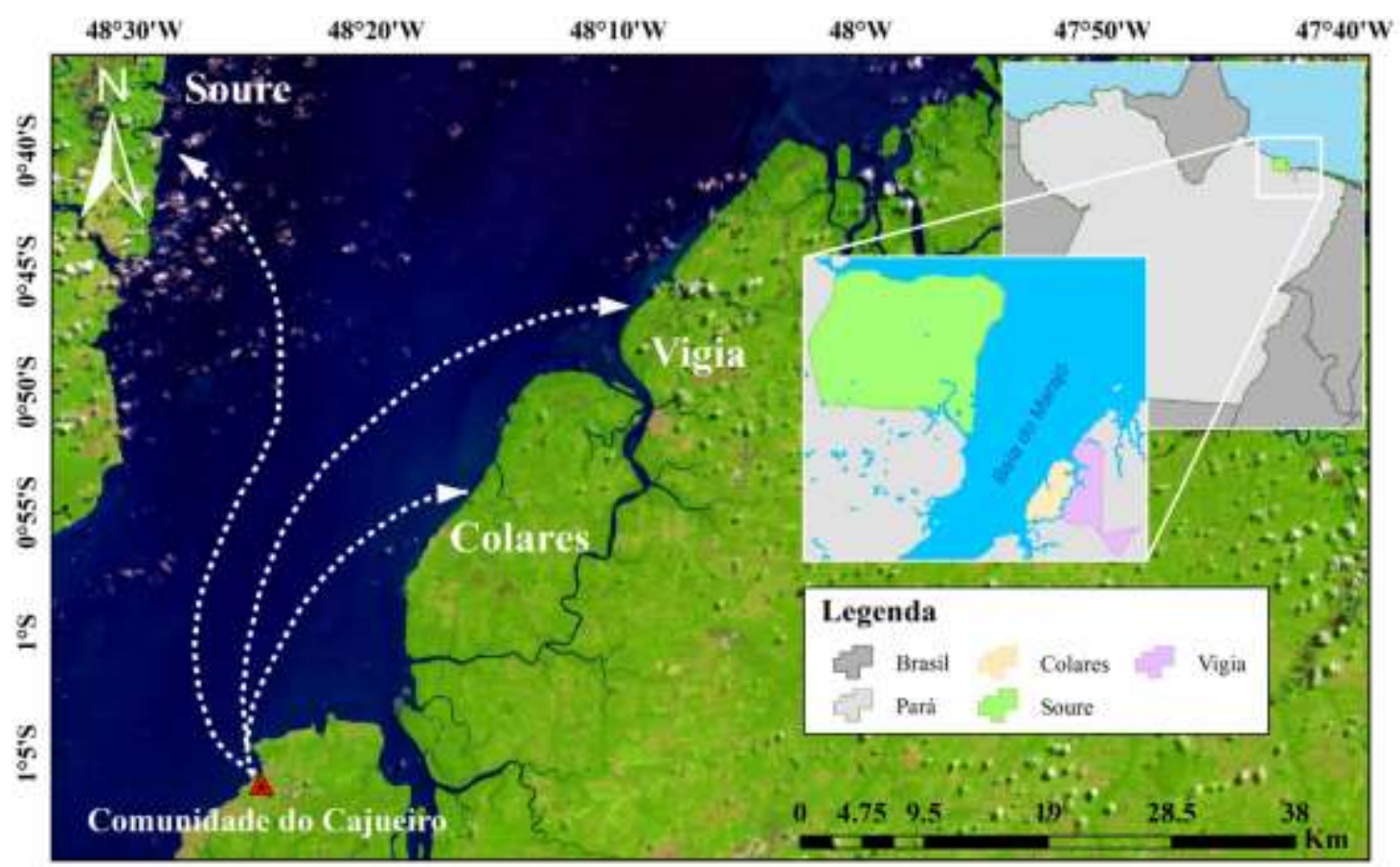

Fonte: Autores (2021).

Os pescadores entrevistados relataram que a cada ano que passa aumenta o deslocamento para pesqueiros mais distantes, devido à baixa captura do peixe na região em que costumam pescar, diminuindo a produção, este fator pode estar relacionado ao aumento do esforço pesqueiro de acordo com o relatado em conversa informal: "Antigamente a gente saia e voltava pra casa cheio de peixe, mas nos últimos dois anos as vezes dá muito fraco e temos que passar mais tempo pescando"; "Onde a gente costumava ir pescar está tendo muito barco, com redes maiores"; "Tem cara que usa uma malha menor e pega peixe miúdo".

O conhecimento dos pescadores sobre o comportamento das espécies capturadas demonstra a relação com a percepção nas mudanças sazonais bem como o comportamento ecológico das espécies. As áreas de captura, são pontos estratégicos, reconhecidos pelos pescadores de acordo com as características das espécies alvo, como ambientes de alimentação, desova ou abrigo (Begossi, 2004; Camargo \& Petrere Jr., 2004). Em relação as dificuldades de competir pela captura, um dos problemas enfrentados por estes pescadores no momento da pescaria é com relação ao aumento de embarcações e com o tamanho de abertura da malha, que muitos pescadores estão utilizando aberturas menores, estas redes são utilizadas principalmente na captura de espécies pelágicas e demersais, sendo consideradas um apetrecho de pesca seletivo.

As informações referentes a relação da abertura de malha e a captura dos peixes mostram-se consistentes com os dados encontrados na literatura sobre a seletividade da rede de emalhe e o tamanho do indivíduo, onde o conhecimento da seletividade referente aos apetrechos de captura permitem a orientação no controle e regulamentação da pesca, visando reduzir a captura e a mortalidade por pesca de peixes jovens, que ameaça os recursos pesqueiros, podendo comprometer o equilíbrio natural das espécies exploradas (Puzzi \& Silva, 1981; Nakatani et al., 1991)

Existem diversas estratégias tradicionais de manejo da pesca aplicadas pelas comunidades, Doria et al. (2008) ressalta que nas pescarias, o convívio diário do pescador com o ambiente aquático somado a necessidade de exploração, o qual é capaz de reconhecer com e ciência a distribuição das espécies exploradas, as variações sazonais na abundância e os movimentos migratórios. 
A pesca artesanal fornece uma importante fonte de alimento e renda para comunidades pesqueiras, embora vários impactos antropogênicos ameace a sustentabilidade ambiental, econômica e sociocultural dessa prática de pesca, há evidências da pesca artesanal influenciar a distribuição e abundância das espécies capturadas afetando a biodiversidade, biomassa, estrutura da assembleia, dinâmica da comunidade e funcionamento do ecossistema (Batista et.al. 2014).

As principais espécies capturadas, de acordo com as entrevistas que apresentam maior valor comercial são: a dourada, Piramutaba, o filhote e a pescada-branca, as espécies que compõe a fauna acompanhante, ou seja, não é alvo da pescaria, apresentam pouco interesse comercial, normalmente são utilizadas para o próprio consumo e outras são descartadas (Tabela 1).

Tabela 1. Lista das espécies capturadas na pesca artesanal pela comunidade do Cajueiro, Mosqueiro, Pará-Brasil.

\begin{tabular}{|c|c|c|}
\hline FAMILIA/ ESPÉCIE & NOME COMUM & CATEGORIA \\
\hline \multicolumn{3}{|l|}{ ARIIDAE } \\
\hline Arius herzbergii (Bloch,1794) & Bagre & Descartado \\
\hline Bagre bagre (Linnaeus, 1766) & Bandeirado & Consumo \\
\hline \multicolumn{3}{|l|}{ ASPREDINIDAE } \\
\hline Aspredinichthys sp. & Rebeca & Descartado \\
\hline \multicolumn{3}{|l|}{ DASYATIDAE } \\
\hline Dasyatis sp. & Arraia & Consumo \\
\hline \multicolumn{3}{|l|}{ DORADIDAE } \\
\hline Lithodoras dorsalis (Valenciennes, 1840) & Bacu & Descartado \\
\hline \multicolumn{3}{|l|}{ LORICARIIDAE } \\
\hline Hypostomus plecostomus (Linnaeus, 1758) & Acari & Consumo \\
\hline \multicolumn{3}{|l|}{ MUGILIDAE } \\
\hline Mugil curema Valenciennes, 1836 & Tainha & Alvo \\
\hline \multicolumn{3}{|l|}{ PIMELODIDAE } \\
\hline Brachyplatystoma rousseauxi (Castelnau, 1855) & Dourada & Alvo \\
\hline Brachyplatystoma filamentosum (Lichtenstein, 1819) & Filhote & Alvo \\
\hline Hypophthalmus marginatus Valenciennes, 1840 & Mapará & Alvo \\
\hline Brachyplatystoma vaillanti (Valenciennes, 1840) & Piramutaba & Alvo \\
\hline Pimelodus blochii Valenciennes, 1840 & Piaba & Consumo \\
\hline \multicolumn{3}{|l|}{ PRISTIGASTERIDAE } \\
\hline Pellona flavipinnis (Valenciennes, 1837) & Sarda & Consumo \\
\hline \multicolumn{3}{|l|}{ SCIAENIDAE } \\
\hline Plagioscion squamosissimus (Heckel, 1840) & Pescada branca & Alvo \\
\hline
\end{tabular}

Fonte: Autores.

Segundo os pescadores da comunidade do Cajueiro, a identificação da presença dos cardumes de dourada e piramutaba é através da observação de uma camada de "resina" ou "gordura" formada na superfície da água: "a água fica com uma gordura por cima, quando tiver isso pode lançar a rede, que ali tem peixe”. De acordo com os relatos a presença dessas espécies está relacionada a ocorrência de cardumes de peixes menores, como as sardinhas que são predados pelas espécies 
alvo: "Quando dá peixe pequeno, pode jogar a rede, que elas vêm comer tudinho" fazendo referência ao habito alimentar da dourada e piramutada.

A dourada encontra-se distribuída em toda a Bacia Amazônia, seguindo, apresenta preferência por ambiente de águas brancas, e pode alcançar um comprimento de $130 \mathrm{~cm}$ e pesar $50 \mathrm{~kg}$, sendo considerados maturos com $10 \mathrm{~kg}$, é uma das espécies com a maior migração já descrita inteiramente dentro de água doce (Santos et al., 2006; Duponchelle et al., 2016; Barthem et al., 2017). A pesca da dourada apresenta padrão sazonal associado ao ciclo hidrológico, no qual os meses de redução do nível do rio correspondem ao período de safra, os cardumes são capturados principalmente de agosto a outubro (Barthem \& Goulding, 2007; Cruz et al., 2017).

A Piramutaba é uma espécie que apresenta ampla distribuição com ocorrência desde a bacia do Orinoco, na Venezuela, até a bacia do rio Parnaíba. A apresenta hábito alimentar carnívoro, alimentando-se de peixes menores (Chaves et al., 2003).

A pescada branca, segundo os relatos, possuí como característica pequenas migrações de acordo com a variação de salinidade na área estuarina. A espécie possui ampla distribuição na América do Sul, incluindo a bacia e estuário Amazônico, destacando a baía do Marajó e a baía do Guajará, com hábito bento-pelágico e alimenta-se basicamente de peixes e crustáceos, ocasionalmente consomem insetos (Barthem, 1985; Santos et. al., 2006).

No que diz respeito às espécies não alvo das pescarias, o bacu e a rebeca apesar de serem abundantes nas capturas, normalmente são descartadas devido ao seu tamanho reduzido. As capturas acidentais podem variar consideravelmente, dependendo do apetrecho e da intensidade de esforço de pesca utilizado, essas espécies são encontradas principalmente em estágio de juvenil e/ou de baixo porte (Catchpole et al., 2011; Brito, 2012; Carvalho et al.,2019).

\section{Considerações Finais}

A comunidade de Cajueiro tem o pescado como um dos principais recursos explorados para comercialização e subsistência, além disso a pesca permite a inclusão de pessoas de diferentes faixas etárias e graus de escolaridade, apesar dessa inclusão social, as mulheres apresentam pouca participação em embarcações ou atuações diretas na captura, apresentando como função principal o beneficiamento, comercialização do pescado, bem como, a confecção e ajustes de apetrechos.

Os pescadores adquiriram conhecimento das espécies, esse conhecimento gerado envolve os hábitos alimentares, migrações, período reprodutivo e locais de captura. Mas com o esforço gerado em cima dos estoques, os pescadores notaram que as espécies capturadas tiveram uma diminuição do tamanho, além disso, precisam fazer maiores deslocamentos para a captura do pescado, tornando a pescaria mais ostensiva.

Essas informações são uteis para o desenvolvimento de estratégias e planos de governo nos espectros ambientais e sociais, visto que é necessário um plano de manejo para as espécies capturadas devido ao intenso esforço de pesca. Ressalta-se que, para o âmbito social é necessário traçar metas para o desenvolvimento escolar, visando qualificar mais os pescadores para auxiliar no aperfeiçoamento da mão de obra na região, além de promover o incentivo para a valorização das mulheres na pesca.

\section{Referências}

Alencar, C. A. G., \& Maia, L. P. (2011). Perfil Socioeconômico dos pescadores brasileiros. Arquivos Ciências do Mar, 44(3), 12-19.

Araújo, I. X., Lima, E. R. V., \& Sassi, R. (2015). Economia da pesca artesanal: O caso do bairro da Penha em João Pessoa/PB. Revista Formação, 1(22),193220.

Bahuchet, S. (2017). Diversidade cultural dos pigmeus africanos. In: Caçadores-coletores da Bacia do Congo. Routledge. p. 1-30.

Bailey, K. D. (1982). Methods of Social Research. Free Press, 553 p. 
Barthem, R. B. (1985). Ocorrência, distribuição e biologia dos peixes da Baía do Marajó, Estuário Amazônico. Boletim do Museu Paraense Emílio Goeldi, série Antropologia, Belém, 6(1), 15-28.

Barthem, R. B., \& Goulding, M. (2007) An unexpected ecosystem: the Amazon revealed by the fisheries. Gráfica Biblos: Lima.

Barthem, R, Goulding M, Leite, R. G., Cañas C, Forsberg, E. V., Petry, P., Ribeiro, M. L. B., Chuctaya, J., \& Mercado A. (2017). Goliath catfish spawning in the far western Amazon confirmed by the distribution of mature adults, drifting larvae and migrating juveniles. Scientific Reports. 7. 101038/srep41784

Batista, V. S., Fabré, N. N., Malhado, A. C., \& Ladle, R. J. (2014). Tropical artisanal coastal fisheries: challenges and future directions. Reviews in Fisheries Science \& Aquaculture, 22(1), 1-15.

Begossi, A. (2004). Áreas, Pontos de pesca, Pesqueiros e Territórios na pesca artesanal, in Ecologia de pescadores da Mata Atlântica e da Amazônia. Ed. (org.) A. Begossi, São Paulo. Hucitec: Nepam/Unicamp: Nupaub/ USP: Fapesp. 223-254.

Begossi, A. (2010). Small-scale fisheries in Latin America: management models and challenges. Mast, 9(2), 7-31.

Borcem, E. R., Furtado Júnior, I., Almeida, I. C., Palheta, M. K. S., \& Pinto, I. A. A. (2011). Atividade pesqueira no município de Marapanim-Pará, Brasil. Revista de Ciências Agrárias, 54 (3), 189-201.

Braga, T. M. P., \& Rebêlo, G. H. (2017). Traditional knowledge of the fishermen of the Lower Juruá River: understanding the reproductive patterns of the region's fish species. Desenvolvimento e Meio Ambiente 40:385-397. http://dx..org/10.5380/dma.v40i0.44776.

Brito, T. P. (2012). O conhecimento ecológico local e a interação de botos com a pesca no litoral do estado do Pará, região Norte - Brasil. Revista Biotemas 260-263.

Camargo, S. A. F., \& Petrere Jr., M. (2004). Análise de risco aplicada ao manejo precaucionário das pescarias artesanais na região do reservatório da UHETucuruí (Pará, Brasil). Acta Amaz 34(3): 473-485.

Carpenter, K. E., \& Niem, V. H. (1999). Fao: species identification guide for fishery purposes. The living marine resources of the western central pacific. V. 3. Batoid Fishes. Chimaeras and bony fishes part. I, Fao, Rome1397-2068.

Carvalho, A. R., Pennino, M. G., Bellido, J. M., \& Olavo, G. (2019). Small-scale shrimp fisheries bycatch: A multi-criteria approach for data-scarse situations. Marine Policy, 10(3): 6-13.

Catchpole, T. L., Enever, R., Maxwell, D. L., Armstrong, M. J., Reese, A., \& Revill, A. S. (2011). Constructing indices to detect temporal trends in discarding. Fisheries Research, 107(1-3), 94-99.

Chaves, R. Silva, K. C. A., Ivo, C. T. C., Cintra, I. H. A., \& Aviz, J. S. (2003). Sobre a pesca da piramutaba, Brachyplatystoma vaillantii (Valenciennes, 1940) em pescarias da frota industrial no estado do Pará. Boletim Técnico-Científico do CEPNOR, 3(1), 163-177.

Cruz, R. E. A., Isaac, V. J., \& Paes, E. T. A. (2017). Pesca da dourada Brachyplatystoma rousseauxii (Castelnau, 1855) na região do baixo amazonas, Brasil. Boletim do Instituto de Pesca de São Paulo, 43(4), 474-486.

Doria, C. R. C., \& Araújo, T. R., Souza, S. T. B., \& Torrente-Vilara, G. (2008). Contribuição da etnoictiologia à análise da legislação pesqueira referente ao defeso de espécies de peixes de interesse comercial no oeste da Amazônia Brasileira, rio Guaporé, Rondônia, Brasil. Biotemas, 21(2), 119-132.

Duponchelle, F., Pouilly, M., Pecheyran, C., Hauser, M., Renno, J. F., Panfili, J., Darnaude, A. M., Garcia-Vasquez, A., Carvajal-Vallejos, F., Garcia-Davila, C., Doria, C., Berail, S., Donard, A., Sondag, F., Santos, R. V., Nuñez, J., Point, D., Labonne, M., Baras, E. (2016). Trans-Amazonian natal homing in giant catfish. Journal of Applied Ecology. 53: 1511-1520.

Espirito-Santo, R. V., Isaac, V. J., Abdon, S., Luis, M., Martinelli, J. M, Higuchi, H, \& Saint-Paul, U. (2005). Peixes e camarões do litoral bragantino ParáBrasil. Belém: MADAM, 268 p.

Fonseca, M, Alves, F., Macedo, M. C, \& Azeiteiro, U. M. (2016). O Papel das Mulheres na Pesca Artesanal Marinha: Estudo de uma Comunidade Pesqueira no Município de Rio das Ostras, RJ, Brasil. Revista de Gestão Costeira Integrad [online], 16(2): 11p.

Froese, R, \& Pauly, D. (2019). Fishbase. In: Species 2000; Itis Catalogue of Life, 2019 Annual Checklist (Roskov, Y, Ower, G, Orrell, T, Nicolson, D, Bailly, N, Kirk, P. M, Bourgoin, T, DeWalt, R. E, Decock, W, Nieukerken, E, Zarucchi, J, \& Penev L, eds.).

Garcez, D. S, \& Shanchez-Botero, J. I. (2005). Comunidades de pescadores artesanais no Estado do Rio Grande do Sul, Brasil. Revista Atlântica, Rio Grande, $27(1), 17-29$.

Maneschy, M. C, Siqueira, D, \& Álvares, M. L. M. (2012). Pescadoras: subordinação de gênero e empoderamento. Revista Estudos Feministas (UFSC. Impresso), 20: 713-737.

Marques, J. G. W. (1995) Pescando Pescadores. Etnoecologia abrangente no baixo São Francisco. NUPAUB-USP. São Paulo, Brasil. 285 pp.

Martorano, L. G. (1993). Estudos climáticos do estado do Pará, classificação climática (Köppen) e deficiência hídrica (Thornthwhite, Mather). Belém: SUDAM/EMBRAPA. 53 p.

McQueen, D. J, Johannes, M. R. S, Post, J. R, Stewart, T. J, \& Lean, D. R. S. (1989), Bottom - Up and Top - Down Impacts on Freshwater Pelagic Community Structure. Ecological Monographs, 59: 289-309.

Mourão, J. S, \& Nordi, N. (2006). Pescadores, peixes, espaço e tempo: uma abordagem etnoecológica Interciencia. Asociación Interciencia. 31(5): 358-363

Nakatani, K, Gomes, L. C, \& Latini, J. D. (1991). Seletividade em redes de espera para captura de Trachydoras paraguayensis (Osteichthyes, Siluriformes), no reservatório de Itaipu e áreas de sua influência. Revista UNIMAR, Maringá, 13(2): 327-338. 
Research, Society and Development, v. 10, n. 2, e12410212336, 2021

(CC BY 4.0) | ISSN 2525-3409 | DOI: http://dx.doi.org/10.33448/rsd-v10i2.12336

Nunes, S C., Pinon, S. H., Maciel, V. T., Barra, L. J, \& Palheta, S. J. M. (2017). Modo de vida e territorialidades de pescadores da comunidade Cajueiro em Mosqueiro (Belém-Amazônia-Brasil). Revista NERA, 20(40).

Pereira, A. S., Shitsuka, D. M., Parreira, F. J., \& Shitsuka, R. (2018). Metodologia da Pesquisa Científica. UAB/NTE/UFSM.

Prodanov, C. C, \& Freitas, E. C. (2013). Metodologia do trabalho científico: métodos e técnicas da pesquisa e do trabalho científico. Novo Hamburgo: Feevale, $277 \mathrm{p}$

Puzzi, A. E., Silva, M. R. G. A. (1981). Seletividade em redes de emalhar e dimensionamento do tamanho de malha para a captura da corvina Micropogonias furnieri (Desmarest, 1823). Boletim do Instituto de Pesca, São Paulo, 8(único): 139-156.

Ramires, M, Clauzet, M, Rotundo, M. M, \& Begossi, A. A. (2012). Pesca e os pescadores artesanais de Ilhabela (SP), Brasil. Boletim do Instituto de Pesca, São Paulo, 38 (3), 231-246.

Sales, G. M, Silva Borges, M, Pereira, J. L. G, Thalês, M. C, \& Almeida, A. S. (2018). Paisagem cultural da Ilha do Mosqueiro: Evolução espaço-temporal (1986-2016). Caminhos de Geografia, 19(65), 204-217.

Santos, G. M, \& Santos, A. C. M. (2005). Sustentabilidade da pesca na Amazônia. Estudos Avançados. 19(54):165-82.

Santos, G. M, Ferreira, E. J. G., \& Zuanon, J. A. S. (2006). Peixes comerciais de Manaus. IBAMA/ProVárzea. 144 p.

Santos, RF, Santos, WJP, Monteiro, EP, \& Nascimento, JCS. (2018). A pesca artesanal no nordeste paraense, município de Viseu-Pará. Acta of Fisheries and Aquatic Resources, 6(1), 35-42.

Silva, J. T, \& Braga, T. M. P. (2016). Caracterização da pesca na comunidade de Surucuá (Resex Tapajós Arapiuns). Biota Amazônia 6(3):55-62. http://dx..org/10.18561/2179-5746/biotaamazonia. v6n3p55-62.

Walter, T, Wilkinson, J., \& Silva, P. A. (2012). A análise da cadeia produtiva dos catados como subsídio à gestão costeira: as ameaças ao trabalho das mulheres nos manguezais e estuários no Brasil. Revista da Gestão Costeira Integrada / Journal of Integrated Coastal Zone Management, $12(4): 483-497$. $10.5894 / \mathrm{rgci} 346$ 